\title{
ChemComm
}

\section{Polymorphism Control of an Active Pharmaceutical Ingredient Beneath Calixarene-based Langmuir Monolayers}

\begin{tabular}{|r|l|}
\hline Journal: & ChemComm \\
\hline Manuscript ID: & CC-COM-02-2014-000928.R1 \\
\hline Article Type: & Communication \\
\hline Date Submitted by the Author: & $19-$-Feb-2014 \\
\hline Complete List of Authors: & $\begin{array}{l}\text { Tulli, Ludovico; University of Applied Sciences Northwestern Switzerland, } \\
\text { School of Life Sciences, Institute of Chemistry and Bioanalytics } \\
\text { Moridi, Negar; University of Applied Sciences Northwestern Switzerland, } \\
\text { School of Life Sciences, Institute of Chemistry and Bioanalytics } \\
\text { Wang, Wenjie; Ames Laboratory, } \\
\text { Helttunen, Kaisa; University of Jyväskylä, Department of Chemistry, } \\
\text { Nanoscience Center } \\
\text { Neuburger, Markus; Universität Basel, Department für Chemistry } \\
\text { Vaknin, David; Ames Laboratory, } \\
\text { Meier, Wolfgang; University of Basel, Institute of Physical Chemistry } \\
\text { Shahgaldian, Patrick; University of Applied Sciences Northwestern } \\
\text { Switzerland, School of Life Sciences, Institute of Chemistry and } \\
\text { Bioanalytics }\end{array}$ \\
\hline
\end{tabular}




\title{
Polymorphism Control of an Active Pharmaceutical Ingredient Beneath Calixarene-based Langmuir Monolayers
}

\author{
Ludovico G. Tulli, ${ }^{a}$ Negar Moridi, ${ }^{a}$ Wenjie Wang, ${ }^{b}$ Kaisa Helttunen, ${ }^{c}$ Markus Neuburger, ${ }^{d}$ David \\ Vaknin, ${ }^{b}$ Wolfgang Meier, ${ }^{d}$ and Patrick Shahgaldian ${ }^{* a}$ \\ 5 Received (in $X X X, X X X)$ Xth $X X X X X X X X X 20 X X$, Accepted $X$ th $X X X X X X X X X 20 X X$
DOI: 10.1039/b000000x
}

This communication demonstrates the possibility to nucleate and grow different crystalline polymorphic forms of gabapentin (GBP) using, as nucleation templates, Langmuir 10 monolayers of an amphiphilic calixarene at different packing densities.

Solid-state polymorphism has a pivotal role in pharmaceutical sciences as many active pharmaceutical ingredients (APIs) exist in several crystalline polymorphic forms, which may exhibit 15 divergent bioavailability and pharmacokinetic properties. ${ }^{1}$ Therefore, the US Federal Drug Administration (FDA) acknowledges each polymorph of an API as a patentable entity. ${ }^{2}$ Besides strategies based on the modulation of chemical (e.g., solvent, co-solvent, additive) and physical (e.g., evaporation, 20 sublimation, thermal treatment, pressure) crystallization conditions, ${ }^{3}$ heterogeneous nucleation represents a valuable alternative to control crystal growth processes with a possible control over the polymorphism of the produced crystals. For example, polymorphism control can be achieved using 25 crystallization templates such as supramolecular gels, ${ }^{4}$ polymer surfaces, ${ }^{5}$ single crystal faces, ${ }^{6}$ self-assembled monolayers ${ }^{7}$ and Langmuir films. ${ }^{8}$ Considering the latter approach, Mann demonstrated that Langmuir monolayers can act as crystal nucleation templates of calcium carbonate in the vaterite 30 polymorphic form. ${ }^{8}$ Besides systems based on simple surfactants, the potential of more complex amphiphiles has also been evaluated. ${ }^{9}$ Recently, we demonstrated that the crystallization kinetics of acetaminophen can be modulated using Langmuir monolayers of an amphiphilic calix[4]arene. ${ }^{10}$ To our knowledge, 35 no control over the polymorphism of APIs through the modulation of the packing of the monolayer has been demonstrated to date. This can be explained by the fact that even if the Langmuir monolayer approach theoretically provides the possibility to control the packing density of template amphiphiles 40 at the air-water interface, strong intermolecular interactions among these molecules often dominate the interfacial selfassembly process. Consequently, this leads to interfacial molecular organizations where the packing state variations are limited. We and others demonstrated that Langmuir monolayers 45 of tetra-dodecyl-p-carboxy-calix[4]arene, during the film compression, undergo an organizational phase transition. ${ }^{10 \mathrm{~b}, 11} \mathrm{In}$ this communication, we demonstrate that this phenomenon can be exploited to control the crystalline polymorphism of gabapentin. 5,11,17,23-tetra-carboxy-25,26,27,28-tetradodecyloxycalix-

50 [4]arene, 1, in the cone conformation, was synthesized as previously described; ${ }^{10 \mathrm{~b}} c f$. Fig. 1 . 1 was recrystallized from pyridine; the resulting weakly diffracting crystals were of suitable quality for single crystal X-ray diffraction studies. Structure was solved in a triclinic space group $P-1$ with one molecule of $\mathbf{1}$ and 55 four molecules of pyridine in the asymmetric unit (ASU); $c f$. Fig. 1 and $\mathrm{S} 1$ in ESI. $\uparrow \mathbf{1}$ is seen to adopt a pinched cone conformation with the shorter arene-arene distance of $4.7 \AA$; this is in agreement with the tetra-butoxy- $p$-carboxylatocalix[4]arene structure reported by Dalgarno. ${ }^{12}$ Symmetry expansion of the ${ }_{60} \mathrm{ASU}$ affords a bilayer structure where the aliphatic chains are organized in an all-parallel fashion, with a slight tilt of $9.7^{\circ}$ with regard to the bilayer axis.
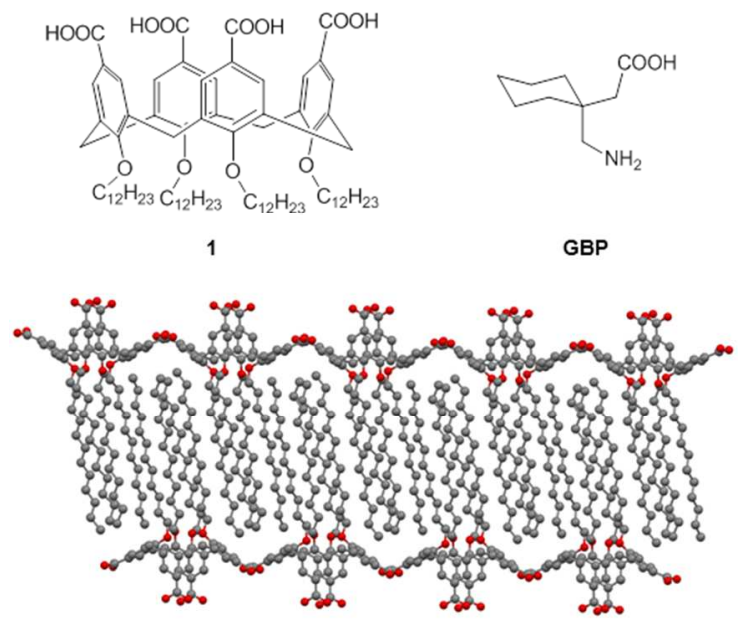

Fig. 1 Chemical structures of 1 and GBP (top) and packing arrangement of the crystal structure of $\mathbf{1}$ (bottom). Solvent molecules and hydrogen 65

$$
\text { atoms are omitted for clarity. }
$$

The interactions of 1, self-assembled as Langmuir monolayers, with GBP were studied by measuring surface pressure-area (П/A) compression isotherms on a GBP subphase $\left(5 \mathrm{~g} \mathrm{~L}^{-1}\right)$; the thickness of the film was measured in real-time during the 70 compression using surface ellipsometry; $c f$. Fig 2. On pure water, the monolayer collapses at an apparent area of $99 \pm 1 \AA^{2}$ molecule $\mathrm{e}^{-1}$ and a surface pressure of $51.5 \pm 0.5 \mathrm{mN} \mathrm{m}^{-1}$, which is consistent with a calixarene-based amphiphile in the cone conformation. $^{13}$ 

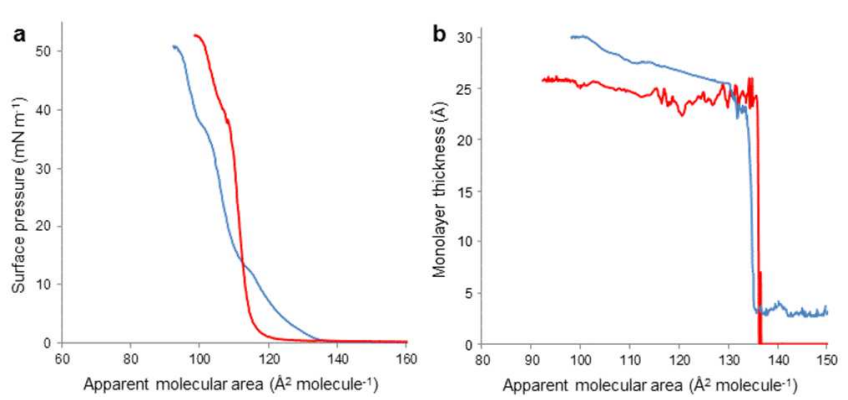

Fig. 2 Surface pressure-area compression isotherms (a) and film thickness measured by surface ellipsometry (b) at the air-water interface of $\mathbf{1}$ on pure water (-) and on a $5 \mathrm{~g} \mathrm{~L}^{-1}$ aqueous GBP solution (-).

The isotherm profile shows a phase transition at s $37.5 \pm 0.5 \mathrm{mN} \mathrm{m}^{-1} \quad\left(107 \pm 1 \AA^{2}\right.$ molecule $\left.^{-1}\right) \quad$ suggesting a molecular rearrangement of the amphiphiles, in agreement with previously reported data. ${ }^{11}$ The ellipsometric measurements reveal that, before reaching an available area per molecule of $137 \pm 0.5 \AA^{2}$ molecule $^{-1}$, no thickness can be measured. This 10 suggests a macroscopic gas-phase behaviour of the amphiphiles. Nevertheless, Brewster angle microscopy experiments show, at 0 $\mathrm{mN} \mathrm{m}^{-1}$, the existence of liquid domains floating at the surface of the water subphase (Fig. S2). Their surface coverage is too low to generate a measurable surface thickness value and the signal 15 remains in the background. Upon further compression, the thickness sharply increases to a value fluctuating around an average of $24 \pm 1 \AA$ and remains constant until the phase transition. After this phase transition, a slight but significant increase of $2 \AA$ is measured and the monolayer thickness reaches ${ }_{20} 26 \pm 1 \AA$. This value is reasonably consistent with the molecular structure of the calixarene macrocycle self-assembled as a densely packed monolayer with the $C_{4}$ pseudo-symmetry axis parallel to the interface. In the presence of GBP, the situation is significantly different. Indeed, even before the isotherm take-off, ${ }_{25}$ an average thickness of $2 \AA$ could be measured, suggesting the presence of islands of liquid phase domains at the interface. At the isotherm take-off $\left(136 \pm 1 \AA^{2}\right.$ molecule $\left.^{-1}\right)$, the thickness sharply increases to $24 \pm 0.5 \AA$ and, beyond this value, steadily increases up to $26 \pm 0.5 \AA$ until the phase transition at $3036 \pm 0.5 \mathrm{mN} \mathrm{m}^{-1}$. Upon further compression, the thickness interestingly increases up to $30 \AA$, a thickness value that is definitely too high to be explained by the presence of only $\mathbf{1}$ at the interface. This higher thickness value can be attributed to a partial layer of GBP in contact with the Langmuir monolayer and 35 thus suggests that the amphiphile organization, after the phase transition, significantly favours the interaction of the monolayer with GBP.

The 2D structure of monolayers of $\mathbf{1}$ was investigated by synchrotron-generated X-ray reflectivity (XR) and grazing 40 incidence X-ray diffraction (GIXD). Inspection of the electron density (ED) profile shows negligible change in the ED of the head group region suggestive of only weak and sporadic adsorption of GBP to the monolayer on a dilute GPB subphase that has no effect on the structure of the calixarene monolayer 45 (Fig. S3). GIXD of the monolayer of 1 ( $c f$. Fig. 3), compressed at $25 \mathrm{mN} \mathrm{m}^{-1}$, shows a broad peak (A1) at $Q_{x y} \sim 1.40 \AA^{-1}$ for $Q_{z}<$ $0.2 \AA^{-1}$ that corresponded to a $d$-spacing $\left(=2 \pi Q_{x y}{ }^{-1}\right) \sim 4.5 \AA$, a feature that was observed for a short chain surfactant on water surfaces $^{14}$ and was attributed to bulk hydrocarbon chains in the

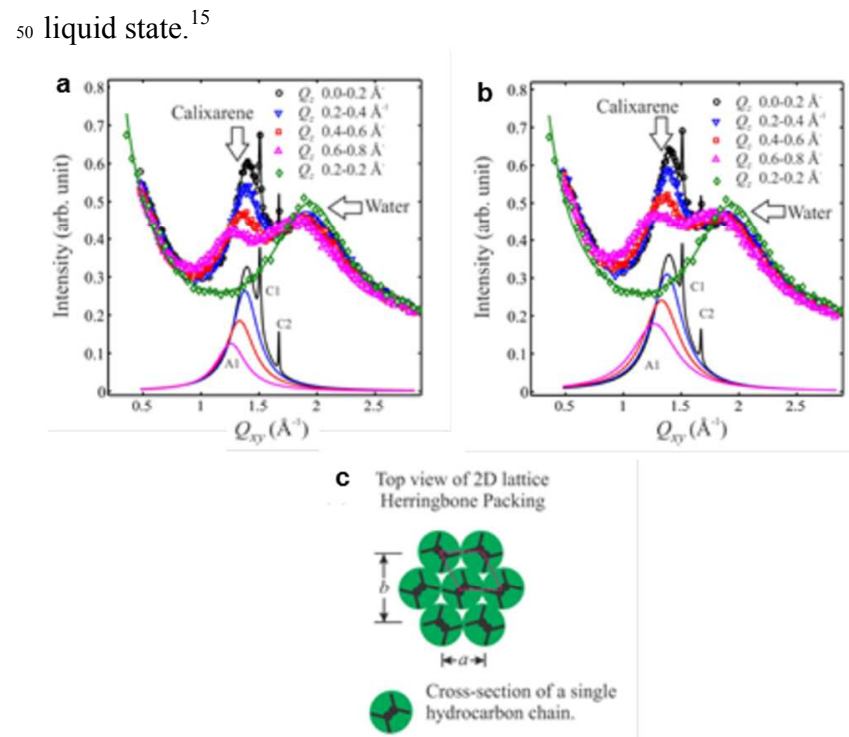

Fig. 3 GIXD patterns of monolayers of $\mathbf{1}$ on water (a) and on a $5 \mathrm{~g} \mathrm{~L}^{-1}$ aqueous GBP subphase (b) both compressed at $45 \mathrm{mN} \mathrm{m}^{-1}$. Each data point is integrated over the $Q_{z}$ range as indicated. The solid lines are the best fit of superposed Lorentzian functions. Solid lines are obtained after 55 subtraction of water signal. Schematic representation of 2D herringbone packing of hydrocarbon chains, in a rectangular unit cell (c). The dashedlines group $4 \mathrm{NN}$ chains that represent a single molecule of $\mathbf{1}$.

Overlay of the A1 peak integrated over different $Q_{z}$ ranges shows that the A1 peak shifts to lower $Q_{x y}$ with increasing $Q_{z}$, while its 60 intensity (over $Q_{x y}$ ) falls off with $Q_{z}$. It is reminiscent of the diffractions from uniaxially oriented polymers, where the chainchain correlations give rise to interference peaks along equatorial directions (i.e. $Q_{x y}$ ) and spread onto an arc toward meridian direction (i.e. $Q_{z}$ ) due to misorientation. The finite extension of ${ }_{65}$ intensity distribution may result from combined effect of finite length of hydrocarbon chains and finite degree of misorientation. The full width at half maximum (FWHM) of A1 (of Lorentzian line shape) is $Q_{x y} \sim 0.2-0.3 \AA^{-1}$, corresponding the lateral short range order (SRO) correlation length $1 \sim 6-10 \AA$, so that A1 peak 70 results from interference of hydrocarbon chains with nearest neighbour $(\mathrm{NN})$ and at most with next nearest neighbour (NNN) chains. When the monolayer is compressed above the phase transition $\left(\pi=45 \mathrm{mN} \mathrm{m}^{-1}\right)$, two additional sharp Braggreflections (C1 and C2) emerge within $Q_{z}<0.2 \AA^{-1}$ at $Q_{x y} \sim 1.51$ 75 and $1.67 \AA^{-1}$, with a peak height ratio $2: 1$. Using a rectangular unit cell with $a=5.0 \AA$ and $b=7.5 \AA$ (Fig. $3 \mathrm{c}$ ), the $\mathrm{C} 1$ and $\mathrm{C} 2$ peaks index as $\{11\}$ and $\{02\}$ crystalline planes, respectively, with $d_{02}=3.76 \AA$ and $d_{11}=4.16 \AA$. This structure is consistent with the herringbone acyl-chain packing commonly observed in ${ }_{80}$ crystalline alkanes and in a few Langmuir monolayers of lipids. ${ }^{16}$ Consequently, this highly ordered phase coexists with SRO previously discussed above the phase transition. Interestingly, although molecules of $\mathbf{1}$ possess a higher degree of molecular complexity compared to alkanes (i.e., four alkyl chains per single 85 molecule), they show similar packing features when selfassembled as Langmuir monolayers. The GIXD pattern of the monolayer of $\mathbf{1}$ on a dilute aqueous GBP subphase $\left(5 \mathrm{~g} \mathrm{~L}^{-1}\right)$ is similar to that on pure water, consistent with the reflectivity that showed a weak influence of GBP on the organization of the 90 monolayer. This strongly suggests that the presence of GBP does 
not drastically influence the hydrocarbon-chains packing of $\mathbf{1}$ at the air-water interface.

The ability of 1-based monolayers to crystallize GBP at the airwater interface was investigated by spreading, at the surface of 5 supersaturated GBP solutions $\left(150 \mathrm{~g} \mathrm{~L}^{-1} \dagger\right)$, adequate amounts of $\mathbf{1}$ corresponding on the Langmuir compression isotherm to values of $0,1,25$ and $45 \mathrm{mN} \mathrm{m}^{-1}$. Interestingly, while the control experiments did not contain any solids, even the vials with a limited amount of $\mathbf{1}$ spread at the interface (corresponding to a 10 surface pressure of $0 \mathrm{mN} \mathrm{m}^{-1}$ ) had crystalline material at the interface after 14 days, a representative photograph is given in Fig. S1. On the other hand, crystals in the control vials appeared as late as after 4 weeks, mostly in solution. Infrared (IR) spectroscopy (Fig. S4) revealed that while the crystals grown 15 beneath monolayers below the surface pressure of the phase transition $\left(0,1\right.$, and $\left.25 \mathrm{mN} \mathrm{m}^{-1}\right)$ were the polymorph $\gamma,{ }^{17}$ those produced above this transition $\left(45 \mathrm{mN} \mathrm{m}^{-1}\right)$ were the polymorph $\alpha ;{ }^{18}$ both structures are anhydrous polymorphs of GBP, and no differences in crystallization kinetics were observed. The hydrate ${ }_{20}$ polymorphic form of GBP, named I, nucleated in the controls vials. ${ }^{18}$ Single crystal X-ray diffraction experiments confirmed the polymorphs identities and face-indexing analyses revealed that, whereas the polymorph a nucleated with the (001) crystallographic plane parallel to the air-water interface, the ${ }_{25}$ polymorph $\gamma$ grew with the (100) crystallographic plane parallel to the interface. Interestingly, both the polymorphs are organized in a bilayered fashion, with the planes of the bilayers parallel to the crystallographic planes. These layered packing supports our initial hypothesis on the possibility to use calixarene-based 30 Langmuir monolayers as crystallization templates where the polar head groups of the amphiphile are expected to act as molecular recognition units, assembling the API in an organized fashion at the interface to initiate crystal growth (cf. Fig. 4).
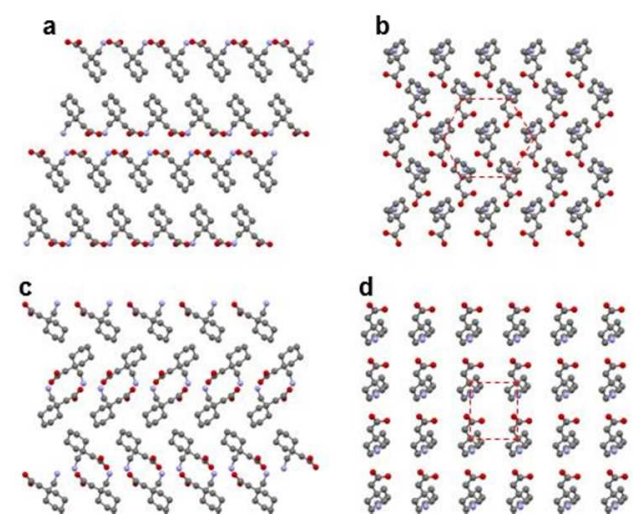

Fig. 4 Packing arrangements of GBP polymorphic form $\gamma$ (a: side view, 35

$$
\text { b: top view) and } \alpha \text { (c: side view, d: top view). }
$$

In our attempts to assess the possibility of epitaxial match between 1-based monolayers and GBP crystals, we applied the model developed by Hillier and Ward that allows studying epitaxial interactions between molecular overlayers and ordered 40 substrates. ${ }^{19}$ The difficulty to conclude from the GIXD data on the molecular organisation of the macrocycle and consequently of the polar recognition headgroups, prevented us from establishing a clear picture of the epitaxial match between the monolayer and the crystals of GBP.
${ }_{45}$ In summary, we have demonstrated that Langmuir monolayers of a calixarene-based amphiphile at different interfacial compression states can trigger the crystallization of two polymorphic forms of GBP ( $\alpha$ and $\gamma$ ). Interestingly, even the phase deprived of longrange crystalline order templates the crystallization of an 50 anhydrous polymorph of the API.

The financial supports of the Swiss Nanoscience Institute (SNI) and the Swiss National Science Foundation (SNSF) are gratefully acknowledged. The work at the Ames Laboratory is supported by the Office of Basic Energy Sciences, U.S. Department of Energy 55 under Contract No. DEAC02- 07CH11358. X-ray diffraction at the Advanced Photon Source is supported by the U.S. Department of Energy, Basic Energy Sciences, Office of Science, under contract No. DE-AC02- 06CH11357.

\section{Notes and references}

${ }_{60}{ }^{a}$ Institute of Chemistry and Bioanalytics, School of Life Science, University of Applied Sciences and Arts Northwestern Switzerland, Muttenz,Switzerland.E-mail: patrick.shahgaldian@fhnw.ch

${ }^{b}$ Ames Laboratory, and Department of Physics and Astronomy, Iowa State University, USA.

${ }_{65}{ }^{b}$ Department of Chemistry, Nanoscience Center University of Jyväskylä, Finland.

${ }^{b}$ Department of Chemistry, University of Basel, Switzerland.

$\dagger$ Electronic Supplementary Information (ESI) available: Experimental details, crystal structures of 1 , photograph of GBP crystals, BAM 70 micrographs, X-ray reflectivity, IR spectra of GBP. See DOI: $10.1039 / \mathrm{b} 000000 \mathrm{x} /$

1 H. G. Brittain, Polymorphism in pharmaceutical solids, Informa Healthcare, 2009.

2 R. Hilfiker, S. M. De Paul and M. Szelagiewicz, in Polymorphism: in 75 the Pharmaceutical Industry, ed. R. Hilfiker, Wiley-VCH, 2006, pp. 287-308.

3 J. Bernstein, in Polymorphism: in the Pharmaceutical Industry, ed. R. Hilfiker, Wiley-VCH 2006, pp. 365-384.

4 J. A. Foster, M.-O. M. Piepenbrock, G. O. Lloyd, N. Clarke, J. A. K.

80 Howard and J. W. Steed, Nat. Chem., 2010, 2, 1037-1043.

5 M. J. Whitcombe, Nat. Chem., 2011, 3, 657-658.

6 C. A. Mitchell, L. Yu and M. D. Ward, J. Am. Chem. Soc., 2001, 123, 10830-10839.

7 C. J. Stephens, Y.-Y. Kim, S. D. Evans, F. C. Meldrum and H. K. 85 Christenson, J. Am. Chem. Soc., 2011, 133, 5210-5213.

8 S. Mann, B. R. Heywood, S. Rajam and D. Birchall, Nature, 1988, 334, 692-695.

9 D. Volkmer, M. Fricke, C. Agena and J. Mattay, J. Mater. Chem., 2004, 14, 2249-2259.

9010 (a) N. Moridi, D. Elend, O. Danylyuk, K. Suwinska and P. Shahgaldian, Langmuir, 2011, 27, 9116-9121; (b) N. Moridi, O. Danylyuk, K. Suwinska and P. Shahgaldian, J. Colloid Interface Sci., 2012, 377, 450-455.

11 M. Strobel, K. Kita-Tokarczyk, A. Taubert, C. Vebert, P. A. Heiney,

95 M. Chami and W. Meier, Adv. Funct. Mater., 2006, 16, 252-259.

12 S. Kennedy, S. J. Teat and S. J. Dalgarno, Dalton Trans., 2012, 39, 384-387.

13 P. Shahgaldian, A. W. Coleman, S. S. Kuduva and M. J. Zaworotko, Chem. Commun., 2005, 1968-1970.

10014 D. Vaknin, S. Dahlke, A. Travesset, G. Nizri and S. Magdassi, Phys. Rev. Lett., 2004, 93, 218302.

15 D. M. Small, The Physical Chemistry of Lipids: From Alkanes to Phospholipids, New York: Plenum Press, 1986.

16 V. M. Kaganer, H. Möhwald and P. Dutta, Rev. Mod. Phys., 1999, $105 \quad 71,779-819$.

17 H. A. Reece and D. C. Levendis, Acta Crystallogr., Sect. C: Cryst. Struct. Commun., 2008, 64, o105-o108.

18 J. A. Ibers, Acta Crystallogr., Sect. C: Cryst. Struct. Commun., 2001, 57, 641-643.

11019 A. C. Hillier and M. D. Ward, Phys. Rev. B, 1996, 54, 14037-14051. 